Conclusions Altogether, our data revealed multiple effects of IL-7 in these patients, highlighting a complex set of in vivo mechanisms. In the future, knowledge of these effects may help in choosing the best agents to use in combination with IL-7 and/or the best patients to benefit from IL-7 as part of their therapeutic approach.

Trial Registration NCT01881867

Ethics Approval The study was last approved by Fred Hutchinson Cancer Research Center Institutional Review Board, IR file 8037, on January 23, 2020

http://dx.doi.org/10.1136/jitc-2020-SITC2020.0263

\section{A PHASE II BASKET TRIAL OF DUAL ANTI-CTLA-4 AND ANTI-PD-1 BLOCKADE IN RARE TUMORS (DART) SWOG S1609: THE THYROID TUMOR COHORT}

\begin{abstract}
${ }^{1}$ Young Kwang Chae, ${ }^{2}$ Megan Othus, ${ }^{3}$ Sandip Patel, 'Sarah Fenton*, ${ }^{4}$ Ding Wang, ${ }^{4}$ Cristina Rodriguez, ${ }^{5}$ Adedayo Onitilo, ${ }^{4}$ Ahmad Mattour, ${ }^{4}$ Igor Rybkin, ${ }^{2}$ Jourdain Hayward, ${ }^{2}$ Christine McLeod, ${ }^{6}$ Helen Chen, ${ }^{6}$ Elad Sharon, ${ }^{2}$ Edward Mayerson, ${ }^{7}$ Christopher Ryan, ${ }^{2}$ Melissa Plets, ${ }^{8}$ Charles Blanke, ${ }^{3}$ Razelle Kurzrock. ${ }^{1}$ Northwestern University, Chicago, IL, USA; ${ }^{2}$ SWOG Statistical and Data Management Cen, Seattle, WA, USA; ${ }^{3}$ University of California at San Diego, La Jolla, California, USA; ${ }^{4}$ Henry Ford Hospital, Detroit, MI, USA; ${ }^{5}$ Marshfield Clinic - Weston Center, Weston, WI, USA; ${ }^{6} \mathrm{NIH}$, Bethesda, MD, USA; ${ }^{7}$ Oregon Health and Science University, Portland, OR, USA; ${ }^{8}$ SWOG Group Chair's Office, Portland, $O R$, USA
\end{abstract}

Background Checkpoint inhibitors acting against PD-1 and CTLA-4 have provided significant benefit for patients. However, their efficacy in rare tumors has not been determined. This abstract presents the results of combination therapy with anti-CTLA and anti-PD-1 in the thyroid cohort of SWOG S1609 Dual Anti-CTLA-4 \& Anti-PD-1 blockade in Rare Tumors (DART).

Methods This study is a prospective, open-label, multicenter phase 2 clinical trial of ipilimumab $(1 \mathrm{mg} / \mathrm{kg}$ intravenously every 6 weeks) plus nivolumab (240 mg intravenously every 2 weeks) in rare tumors. Here we report the outcomes from thyroid cancer patients. The primary endpoint is objective response rate (ORR) (RECIST v1.1) (confirmed complete response (CR) and partial responses (PR)); progression-free survival (PFS), overall survival (OS), stable disease (SD) $>6$ months, and toxicity are secondary endpoints.

Results Twenty-one patients were registered and seventeen were eligible and received therapy. Median age of the patients was 59 (range $33-78), 59 \%(\mathrm{~N}=10)$ of enrolled patients were male. The most common subtype of thyroid cancer was papillary $(47 \%, \mathrm{~N}=8)$, then medullary $(24 \%, \mathrm{~N}=4)$, anaplastic $(24 \%, \mathrm{~N}=4)$ and Hurtle cell histology $(6 \%, \mathrm{~N}=1)$. The ORR was $12 \%$ [CR, $0 \%, \mathrm{~N}=0 ; \mathrm{PR}, 12 \%, \mathrm{~N}=2 ; 1$ papillary and 1 anaplastic subtype]; in addition $12 \%(\mathrm{~N}=2)$ had unconfirmed PR (both papillary subtype). Of note, 1 out of 4 patients with anaplastic thyroid carcinoma (25\%) had a response that lasted more than two years. $35 \%(\mathrm{~N}=6)$ of patients had SD for over 6 months, 12\% (N=2) had SD for less than 6 months (figure $1 \mathrm{a \& b}$ ). Clinical benefit rate including all PRs and SD over 6 months was 59\% ( $\mathrm{N}=10$ / 17). Additionally, one patient with papillary thyroid carcinoma that withdrew early due to toxicities (neuropathic pain and arthralgias) had stable disease for over one year (not included in response assessment). 6-month PFS was 58\% (95\% confidence interval; 39-88) and median PFS was 9.5 months (4.99-infinity); 6-month OS was $88 \%$ (74-100\%) and median OS was not reached. One patient died while enrolled. $94.1 \%$
$(\mathrm{N}=16)$ experienced toxicities with $52.9 \%(\mathrm{~N}=9)$ experiencing grade 3-5 toxicities. The most common adverse events were fatigue $(41.2 \%, \mathrm{~N}=7)$, elevated lipase $(29.4 \%, \mathrm{~N}=5)$, acute kidney injury, diarrhea, muscle weakness, anorexia, pruritis, nausea and alanine aminotransferase elevation $(21.1 \%, \mathrm{~N}=3$ each). The most common immune-mediated adverse events were acute kidney injury and elevated lipase $(29.4 \%, \mathrm{~N}=5$ each).
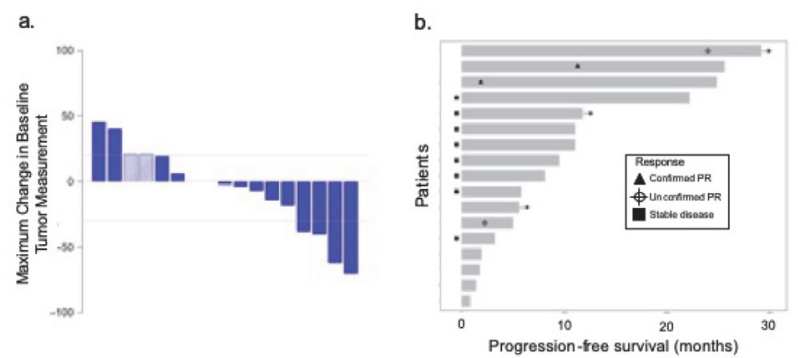

Abstract 270 Figure 1

a. Waterfall plot indicating maximum change in baseline tumor measurement following treatment. Crosshatch indicate patients failed therapy and do not have tumor measurements available due to early clinical progression or progression due to new lesions without RECIST measurable changes $(\mathrm{N}=2)$. b. Swimmer's plot of PFS following therapy. Triangles idicated confirmed PR, targets indicate unconfirmed PR and squares indicate SD.

Conclusions Combination therapy with ipilimumab plus nivolumab in thyroid cancer resulted in an ORR of $12 \%$ with two partial responses in seventeen treated patients.

Trial Registration NCT02834013

Ethics Approval The study was approved by the NCI Adult Central Institutional Review Board, approval number 02834013 .

http://dx.doi.org/10.1136/jitc-2020-SITC2020.0270

\section{CORRELATION OF VIRUS-SPECIFIC CD8+ T CELLS TO CLINICAL RESPONSE FOLLOWING TREATMENT WITH PEXA-VEC AND CEMIPLIMAB IN PATIENTS WITH ADVANCED RENAL CELL CARCINOMA}

${ }^{1}$ Myles Dillon, ${ }^{1}$ Lianjie Li, ${ }^{2}$ Jeongsook Bang, ${ }^{3}$ Nicholas Gaspar, ${ }^{1}$ Jessica Kuhnert, ${ }^{1}$ Nathalie Fiaschi, ${ }^{1}$ Vladimir Jankovic, ${ }^{1}$ Israel Lowy, ${ }^{1}$ Gavin Thurston, ${ }^{1}$ Glenn Kroog, ${ }^{3}$ Kyoung Soo Ha, 'Raquel Deering, 'Raquel Deering*. 'Regeneron Pharmaceuticals, Inc., Tarrytown, NY, USA; ${ }^{2}$ sillajen Inc., Seoul, Korea, Republic of; ${ }^{3}$ Sillajen Biotherapeutics, San Francisco, CA, USA

Background To better understand the immune stimulatory mechanisms of Oncolytic virus (OV), we evaluated the circulating $\mathrm{OV}$-specific $\mathrm{T}$ cell response in patients during the course of OV therapy. Patients with histologically confirmed advanced clear cell renal cell carcinoma, who were naïve or refractory to prior systemic treatment and who had no prior treatment with immune checkpoint inhibitors, were treated with 4 weekly intravenous infusions of Pexa-Vec at $1 \times 10^{9}$ plaque forming units starting at Day -7 plus Cemiplimab $(350 \mathrm{mg}$ every 3 weeks) from Day 1. Radiographic assessments per RECIST 1.1 were performed centrally every 9 weeks from Day 1. Peripheral blood mononuclear cells (PBMCs) were collected and cryopreserved at baseline and 29 days post initial Pexa-Vec treatment. 